\title{
Genome-wide identification and expression analysis of Bcl-2 gene family under low temperature stress in tilapia (Oreochromis niloticus)
}

\author{
Changgeng Yang1, a, Meizi Wang2, a, Hua Wen², Ming Jiang ${ }^{2 *}$, Juan \\ Tian $^{2}$, Xing Lu²
}

${ }^{1}$ Life Science and Technology School, Lingnan Normal University, Zhanjiang, 524048, China

${ }^{2}$ Key Lab of Freshwater Biodiversity Conservation of Ministry of Agriculture and Rural Affairs, Yangtze River Fisheries Research Institute, Chinese Academy of Fishery Sciences, Wuhan 430223, China

Key words: tilapia, $B C l-2$, Phylogeny, low temperature stress, expression profile

\begin{abstract}
Low temperature stress can lead to variety of changes, including apoptosis in tilapia (Oreochromis niloticus). The B cell lymphoma-2 (BCl-2) gene family plays an important role in the process of apoptosis. The present study conducted genome-wide characterization of the $\mathrm{BCl}-2$ family genes in tilapia and their mRNA expression profiles were analyzed in different tissues of tilapia under the low temperature stress $\left(10^{\circ} \mathrm{C}\right)$. Twenty-four $\mathrm{BCl}-2$ family genes were identified, containing 2 8 exons. These genes were classified into two subfamilies ( $\mathrm{BCl}-2$ homologs and $\mathrm{BH} 3-$ only) based on their conserved domains. Besides, these $\mathrm{BCL}-2$ proteins in tilapia possess at least one of the four conserved $\mathrm{BH}$ domains. The phylogenetic analysis showed that the $\mathrm{BCl}-2$ family genes did not aggregate by species, demonstrating sequence conservation of different types of $\mathrm{BCl}-2$ family members. Real-time quantitative PCR (RT-qPCR) analysis showed that $\mathrm{BCl}-2$ family genes were broadly expressed in different tissues of tilapia. When reared at $10^{\circ} \mathrm{C}$, the transcriptional expression levels of most of anti-apoptotic $\mathrm{BCl}-2$ homologs subgroup members and other $\mathrm{BH} 3-$ only subgroup members in most tissues of tilapia were higher than those at $30^{\circ} \mathrm{C}$. However, most of other $\mathrm{BCl}-2$ family members revealed a lower expression. The results suggested that hypothermia had significantly induced apoptotic in tilapia.
\end{abstract}

* Corresponding author. e-mail: jiangming@yfi.ac.cn (Ming Jiang)

a These authors contributed equally to the paper 


\section{Introduction}

Apoptosis is a genetically regulated and programmed cell death, which plays an important role in the growth and development of multicellular organisms and the homeostasis of cell numbers (Renault and Chipuk, 2014). BCl-2 genes family is an important genes family, which functions as important regulators in the apoptotic pathway and participates in the regulation of cell proliferation and autophagy (Levine et al., 2008). It can determine whether the cell apoptosis occurs or not (Adams and Cory, 2018; Danial and Korsmeyer, 2004). The $\mathrm{BCl}-2$ gene, a first discovered $\mathrm{BCl}-2$ family gene, was discovered in human follicular B cell lymphomas more than 20 years ago (Tsujimoto et al., 1984), and its antiapoptotic function was confirmed in 1989 (Tsujimoto, 1989). With the deepening of research, many $\mathrm{BCl}-2$ genes family members were discovered and identified, and could be divided into two functionally opposing subsets: the anti-apoptotic $\mathrm{BCl}-2$ genes and proapoptotic BCl-2 genes (Czabotar et al., 2014). All members in the $\mathrm{BCl}-2$ genes family proteins have at least one of the four conserved $\mathrm{BH}$ domains, and one transmembrane region can be found at the C-terminal of most of $\mathrm{BCL}-2$ family proteins (Christoph, 2003; Lanave et al., 2004). The BH3 domain is known as a death domain, and the detailed mechanism by which it interacts with pro-survival related molecules to regulate apoptosis has become a hot topic in recent years (Strasser et al., 2010; Suzanne et al., 2003). Threedimensional structural studies have shown that the $\mathrm{BH}$ domain is usually required for the formation of dimers between pro-apoptotic proteins and anti-apoptotic proteins (Borner, 2003), and generally the $\mathrm{BH} 1, \mathrm{BH} 2$, and $\mathrm{BH} 3$ domain of anti-apoptotic proteins forms an elongated hydrophobic pocket. The $\mathrm{BH} 3$ domain of the pro-apoptotic protein binds to it as an amphipathic helix, stabilized by the $\mathrm{BH} 4$ domain of the anti-apoptotic protein, which allows the $\mathrm{BH} 3$ domain to be covered and thus unable to exert its apoptotic activity (Sattler et al., 1997). On the reports about the description and classification of $B C l-2$ family genes of Aouacheria(Aouacheria et al., 2013), BCl-2 family genes could be identified and further subdivided into three subfamilies, $\mathrm{BCl}-2$ homologs, canonical $\mathrm{BH}$-only and other $\mathrm{BH}$-only, based on the composition of BH motifs. Moreover, the $\mathrm{BCl}-2$ homologs subfamily had could be divided into three subgroups (pro-apoptotic $\mathrm{BCl}-2$ homologs, anti-apoptotic $\mathrm{BCl}-2$ homologs, and divergent $\mathrm{BCl}-2$ homologs) based on their function and composition of $\mathrm{BH}$ motifs. $\mathrm{BCl}-2$ homologs subfamily had one or more $\mathrm{BH} 1-4$ functional domains. $\mathrm{BH} 3-$ only subfamily had only BH3 functional domains.

Temperature is one of the most important environmental parameters affecting tilapia (Oreochromis niloticus) cultivation. The growth temperature for tilapia is between $16^{\circ} \mathrm{C}$ and $38^{\circ} \mathrm{C}$ (Wohlfarth and Hulata, 1981). If the temperature is lower than $13^{\circ} \mathrm{C}$ or drops rapidly, tilapia will experience the process of adaptation, motion imbalance, stress, coma, and death (Behrends and Smitherman, 2010; Kindle and Whitmore, 2010; Sun et al., 1992), indicating that tilapia has poor cold resistance (Potts et al., 1967). As reported, low-temperature stress can significantly affect the behavior and physiology of tilapia, including a decline in the immune system, changes in physiological characteristics, and potential death (Yang et al., 2015). With the decrease in temperature, the plasma osmotic pressure (Atwood et al., 2015; Sun et al., 1992), serum sodium concentration, and lymphocyte concentration of Nile tilapia decreased, while serum glucose concentration increased (Atwood et al., 2015; Renault and Chipuk, 2014). In addition, there appears to be a series of changes in cellular levels under low temperature conditions, such as changes in cell membrane fluidity and cell mass transport, which can lead to cell division, growth arrest, and apoptosis (Los and Murata, 2004; Yang et al., 2017). Moreover, when subjected to low temperature, the gene expression involved in apoptosis of tilapia is also changed (Yang et al., 2015; Zhou et al., 2018).

In this study, we focused on the apoptosis related gene family, $B C l-2$, to reveal the connection between apoptosis and low-temperature stress. Firstly, we analyzed the gene structures, conserved domains, and phylogenetic relationships of tilapia $\mathrm{BCl}-2$ family genes, which are closely related to apoptosis. Twenty-nine $\mathrm{BCl}-2$ family genes were identified by searching tilapia genome-wide data using bioinformatics methods. The tissue-specific expression analysis of these genes in tilapia was carried out to understand the composition and expression characteristics of $\mathrm{BCl}-2$ family genes. Secondly, the $\mathrm{BCl}-2$ gene expression 
profiles in tilapia reared under low temperature and normal temperature conditions were analyzed. The present study would provide insight into the effect of low-temperature stress on $\mathrm{BCl}-2$ genes expression in tilapia, and lay a foundation for further research on the function of fish $\mathrm{BCl}-2$ genes.

Declaration of Ethics Statement

\section{Materials and Methods}

Tilapia is cultivated widely in South China and is not listed as endangered or protected species. All the experimental animal programs involved in this study were approved by the Yangtze River Fisheries Research Institute's animal care and use committee, and followed the experimental basic principles. The field studies did not involve endangered or protected species.

Experimental fish and sample preparation

Nile tilapia were purchased from a fish hatchery in Guangxi Province of China, and then transported to the experimental base of the Yangtze River Fisheries Research Institute (Wuhan, Hubei Province, China). Their initial body weights were $100.0 \pm 10.0 \mathrm{~g}$. The fish were domesticated for two weeks in an indoor recirculating aquaculture system to allow for a substantial amount of time to accommodate to a new environment. The fish were grown for 1 week at $30^{\circ} \mathrm{C}$, then subjected to low temperature of $10^{\circ} \mathrm{C}$ by cooling the water at a rate of $1^{\circ} \mathrm{C}$ per day ( $\mathrm{Li}$ et al., 2002). Dissolved oxygen was maintained at $>5 \mathrm{mg} / \mathrm{L}$ by an air compressor, water $\mathrm{pH}$ was 7.2-7.5, and the total ammonia nitrogen concentration was $0.26 \pm 0.10 \mathrm{mg} / \mathrm{L}$. One month later, three individuals in normal temperature $\left(30^{\circ} \mathrm{C}\right)$ or low temperature $\left(10^{\circ} \mathrm{C}\right)$ were anesthetized and samples of heart, liver, intestine, muscle, fin, brain, spleen, skin, and gill were collected and frozen in liquid nitrogen. Samples were stored at $-80^{\circ} \mathrm{C}$ until use.

Genome-wide screening and classification of $\mathrm{BCl}-2$ family genes in tilapia

The whole genome sequence of tilapia was downloaded from NCBI database (https://www.ncbi.nlm.nih.gov/). All predicted candidate $\mathrm{BCl}-2$ genes family protein sequences were scanned by PFAM (http://pfam.xfam.org/family/PF00125) and PROSITE (https://prosite.expasy.org/) software for known BCL-2 protein domain/motif. And the genes sequences of all predicted protein were also obtained. Then, using PCR, the genes of all predicted protein were validated. Meanwhile, the obtained genes are classified into subclass of BCL-2 family, according to their function base on original bioinformatics analysis (BLASTP search) in NCBI database. Additional protein domains/motifs may be added by searching conserved domains using Batch CD-Search tool (https://www.ncbi.nlm.nih.gov/Structure/bwrpsb/bwrpSB.cgi).

Molecular evolution analysis of $\mathrm{BCl}-2$ family genes in tilapia

BLASTP searches the known BCL-2 family gene sequences of tilapia, zebrafish (Danio rerio), common carp (Cyprinus carpio) and house mouse (Mus musculus) in the UniProt protein database $(e<1 e-5)$. FASTA file containing zebrafish, common carp, house mouse and tilapia amino acid sequence of $\mathrm{BCl}-2$ family genes were subjected to multiple sequence alignments using ClustalX software. MEGA7.0 software was used to construct a phylogenetic tree using Neighbor-Joining method with Bootstrap value of 1000 . The resulting Newick phylogenetic tree file was visualized by iTOL software (https://itol.embl.de).

Tissue expression of $B C l-2$ family genes in tilapia

Total RNA was extracted from the heart, liver, small intestine, muscle, fin, brain, spleen, skin, and gill tissues of tilapia reared under low temperature and normal temperature conditions, using TRIzol Reagent (Tiangen Biotech, China), respectively. Highquality RNA determined by OD A260/A280 ratio (1.8-2.0) and electrophoresis were subjected to reverse transcription (500ng of total RNA per sample) using FastkinggDNA 
Dispelling Quant RT Kit cDNA (Tiangen Biotech, China). cDNA was used as a template for fluorescence quantitative PCR detection.

RT-qPCR was performed on a QuantStudio 6 Flex Quantitative PCR instrument (Life Technologies, USA). The $20 \mu \mathrm{LqRT}-\mathrm{PCR}$ reaction system consisted of $10 \mu \mathrm{L}$ of $2 \times$ SuperRealPreMix Plus (Tiangen Biotech, China), $6.8 \mu \mathrm{L}$ of RNase-free $\mathrm{ddH} 2 \mathrm{O}, 1.0 \mu \mathrm{L}$ of cDNA template $(500 \mathrm{ng} / \mu \mathrm{L})$, and $0.6 \mu \mathrm{L}$ of forward and reverse primer $(10 \mu \mathrm{mol} / \mathrm{L})$. qRT-PCR reaction conditions are as follows, $95^{\circ} \mathrm{C}$ denaturation $5 \mathrm{~min} ; 28$ cycles include $95^{\circ} \mathrm{C} 30 \mathrm{~s}$, $60^{\circ} \mathrm{C} 30 \mathrm{~s}, 72^{\circ} \mathrm{C} 30 \mathrm{~s} ; 72^{\circ} \mathrm{C} 5 \mathrm{~min}$. Each sample is duplicated 3 times. The relative gene expression was normalized to 18S rRNA (NCBI accession number: JF69868318S) levels using $2^{-\Delta \Delta C T}$ method. The gene-specific primers were designed using the Primer premier software 5 and are listed in Table 1.

Table 1 Primers used for qRT-PCR.

\begin{tabular}{|c|c|c|}
\hline \multirow{2}{*}{ Gene } & \multicolumn{2}{|l|}{ Sequence } \\
\hline & Forward Primer $\left(5^{\prime} \rightarrow 3\right.$ ') & Reverse Primer $\left(5^{\prime} \rightarrow 3^{\prime}\right)$ \\
\hline $\begin{array}{l}\mathrm{Bcl} 2 \mathrm{l} 1 \mathrm{a} \\
(\mathrm{Bcl} 2 \text { like1a) }\end{array}$ & GCATACAATGGCATAGAGGCTTT & GATTCCATCCCTGAACAАСТССТ \\
\hline $\begin{array}{l}\mathrm{Bcl} 2 \mathrm{I} 1 \mathrm{~b} \\
(\mathrm{Bcl} 2 \text { like1b) }\end{array}$ & AATGAGTTCGAGCTGCGATACG & GGAACACCTCGTCCATCACGT \\
\hline Mcl1 & & \\
\hline $\begin{array}{l}\text { (Bcl2 family apoptosis } \\
\text { regulator) }\end{array}$ & ATGACAGAACACAACCACTCGG & CTACAGGCCСTCAAACTCGTAC \\
\hline (Bcl2a & TAACTGACCCTCCACCGACTTT & TCTATCACCTCGGCGAACCTC \\
\hline $\begin{array}{l}\mathrm{Bcl} 2 \mathrm{~b} \\
\text { (Bcl2, apoptosis regulator) }\end{array}$ & AGCGACTTCACGGCAACGAG & GTGACGATGCGTCCCCAGTT \\
\hline $\begin{array}{l}\text { NR13 } \\
(\mathrm{Bcl} 2 \mid 10) \\
\text { Baxa }\end{array}$ & GCTGGTTGGAGATGGACACTTG & TGGCACTGCGGGAGAACTTAC \\
\hline $\begin{array}{l}\text { (Bcl2 associated } \mathrm{x}, \\
\text { apoptosis regulator) }\end{array}$ & AAGATGGCAATAAAGCAGTGACG & TGCAAAGTAGAACAGGGCAACC \\
\hline Baxb & & \\
\hline $\begin{array}{l}\text { (Bcl2 associated } \mathrm{x} \\
\text { apoptosis regulator) }\end{array}$ & TGCAGCATGACCAGGAGTTTC & AAGTTCACTGCCCAGGTAAGGAC \\
\hline Baxc & & \\
\hline $\begin{array}{l}\text { (Bcl2 associated } \mathrm{x} \\
\text { apoptosis regulator) }\end{array}$ & GGAGCGGTTGTCTTTAGAGGGT & TCTGAAGTTCAGCATTCCGATTT \\
\hline $\begin{array}{l}\text { Boka } \\
\text { (Bok, Bcl2 family apoptosis } \\
\text { regulator) }\end{array}$ & ACTGTGTCCGCCATGGTCAT & TCCACCCAGCСTCСТСТСTT \\
\hline Bokb & & \\
\hline $\begin{array}{l}\text { (Bok, } \mathrm{Bcl} 2 \text { family apoptosis } \\
\text { regulator) }\end{array}$ & ATTTTTCTCССTCGAACGCAG & ACATGGACACCACCTTACCCC \\
\hline $\begin{array}{l}\mathrm{Bcl} 2 \mathrm{I} 12 \\
(\mathrm{Bcl} 2 \text { like } 12)\end{array}$ & CACCACAAGTACAGTTCCAACCC & TGTCTTGCCCTTCCTGCCC \\
\hline $\begin{array}{l}\text { bcl2l13 } \\
\text { (Bcl2 like 13) }\end{array}$ & CACCTGTCAGCAGCCGTACAC & ААТАСТТСАССССАТССССС \\
\hline $\begin{array}{l}\mathrm{Bcl} 2114 \\
(\mathrm{Bcl} 2 \text { like } 14)\end{array}$ & TGAGGTTCAGATTCAGAGGCAGAC & ACCCAAGCCGAGTGGTTCTC \\
\hline $\begin{array}{l}\mathrm{Bcl} 2 \mathrm{I} 15 \\
\text { (Bcl2 like 15) }\end{array}$ & AGAACGCTGACATTGCTCCAG & ААСТTТАСТТАСССАТССАССТTТС \\
\hline Bid & & \\
\hline $\begin{array}{l}\text { (BH3 interacting domain } \\
\text { death agonist) } \\
\text { Bad }\end{array}$ & TCAACAGCAATGGGCACGAC & TCAGGTTCCTGGTAGCTTCAGTC \\
\hline $\begin{array}{l}\text { (Bcl2 associated agonist of } \\
\text { cell death) }\end{array}$ & CTTGCCCTTCCTGTAATCAAAAC & GTCAAACTCGTCACTCATCCGTC \\
\hline $\begin{array}{l}\mathrm{Bcl} 2 \mathrm{l} 11 \\
(\mathrm{Bcl} 2 \text { like 11) }\end{array}$ & CCCTCTAGGACGTGGCAGC & CATCGCCCAATCAGTATCAGC \\
\hline $\begin{array}{l}\text { Bmfa } \\
\text { (Bcl2 modifying factor) }\end{array}$ & CAGCCTTGCCTACCGTTTCAT & CTGCCACGCTGTCAATGTCTT \\
\hline $\begin{array}{l}\text { Bmfb } \\
\text { (Bcl2 modifying factor) }\end{array}$ & TCACAGAGCGAGGCGACAAG & TCAAACAGAAGGCTGAGAAGGG \\
\hline
\end{tabular}




\begin{tabular}{|c|c|c|}
\hline $\begin{array}{l}\text { Bnip3a } \\
\text { (Bcl2 interacting protein 3) }\end{array}$ & AAATGCTGACTGGATCTGGGAC & GTATGTGGGAAATGATAAATGATGG \\
\hline $\begin{array}{l}\text { Bnip3b } \\
\text { (Bcl2 protein-interacting } \\
\text { protein 3) }\end{array}$ & GACCCACCAGCGAGCACG & CCAGCAGCGAGGGAACAAG \\
\hline $\begin{array}{l}\text { Bnip3lb } \\
\text { (Bcl2 protein-interacting } \\
\text { protein 3-like) }\end{array}$ & GCCATCGTCСТCСТCTATCCAC & AGTCAGCAACCCAGTCTACTTCTIT \\
\hline $\begin{array}{l}\text { Bnip3la } \\
\text { (Bcl2 protein-interacting } \\
\text { protein 3-like) }\end{array}$ & GGGTGGAGTTGGAGATGAACAG & GGATTGAAGAAGACGATGGGAC \\
\hline $\begin{array}{l}18 \mathrm{~S} \\
\text { (18S ribosomal RNA) }\end{array}$ & GGACACGGAAAGGATTGACAG & GTTCGTTATCGGAATTAACCAGAC \\
\hline
\end{tabular}

\section{Results}

Identification and classification of the $\mathrm{BCl}-2$ family genes in tilapia genome A total of twenty-four $\mathrm{BCl}-2$ genes with conserved $\mathrm{BH}$ domains were identified from the tilapia genome. Meanwhile, we also searched and analyzed the $\mathrm{BCl}-2$ family genes in other two teleost; zebrafish (D. rerio) and common carp (C. carpio), and a mammal, house mouse (M. musculus), with 27, 34 and 35 genes respectively (Table 2 and $\mathbf{3}$ ). Like the description in Abdel (Aouacheria et al., 2013), the $\mathrm{BCl}-2$ family genes were divided into two

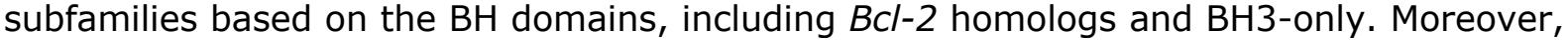
the $\mathrm{BCl}-2$ homologs can be further divided into three subgroups, including pro-apoptotic $\mathrm{BCl}-2$ homologs, anti-apoptotic $\mathrm{BCl}-2$ homologs, and divergent $\mathrm{BCl}-2$ homologs. Among them, Anti-apoptotic $\mathrm{BCl}-2$ homologs have seven members, and their protein structures contain four short conserved $\mathrm{BH}$ domains (BH1-BH4). Pro-apoptotic $\mathrm{BCl}-2$ homologs have five members, and their protein structures contain three to four short conserved $\mathrm{BH}$ domains (BH1-BH4). Divergent $\mathrm{BCl}-2$ homologs have five members with multiple $\mathrm{BH}$ domains. Moreover, the BH3-only subfamily has eight members, and this subfamily member only contained one $\mathrm{BH} 3$ domain or a C-terminal hydrophobic tail structure, i.e., transmembrane (TM) function domain. Among them, canonical $\mathrm{BH}$-only subgroups have 4 members, and these subfamily members only contained one BH3 domain. The other $\mathrm{BH} 3$-only subgroups have 4 members who had one $\mathrm{BH} 3$ domain and a TM function domain. The results showed that the longest $\mathrm{BCL}-2$ protein ( $\mathrm{BCl} 2 \mathrm{l} 13)$ contained 554 amino acids and the shortest one (Bcl2l14) only contained 127 amino acids (Table 4).

Table 2 In Silico identification of $\mathrm{BCl}-2$ family genes in tilapia genome (Oreochromis niloticus genome assembly).

\begin{tabular}{|c|c|c|c|c|c|c|c|c|}
\hline Subg & groups & $\begin{array}{c}\text { Gene } \\
\text { Name } \\
\text { assigne }\end{array}$ & $\begin{array}{c}\text { Gene ID } \\
\text { (NCBI/EMBL) }\end{array}$ & $\begin{array}{c}\text { Chromeso } \\
\text { me }\end{array}$ & Genomic Position & $\begin{array}{l}\text { Exon } \\
\text { Numb }\end{array}$ & Protein_ID & $\begin{array}{c}\text { Lengt } \\
h\end{array}$ \\
\hline & & $\mathrm{Bcl} 2 \mathrm{I} 1 \mathrm{a}$ & 100704382 & LG20 & $\begin{array}{c}31801604 . .3183 \\
5473\end{array}$ & 3 & $\begin{array}{c}\text { XP_0034570 } \\
09.1\end{array}$ & 232 \\
\hline & & $\mathrm{Bcl} 2 \mathrm{I} 1 \mathrm{~b}$ & 100700270 & LG5 & $\begin{array}{c}31247734 . .3124 \\
9724\end{array}$ & 3 & $\begin{array}{c}X P \_0034427 \\
85.1\end{array}$ & 197 \\
\hline & $\begin{array}{l}\text { Anti- } \\
\text { apoptotic }\end{array}$ & Mcl1 & 100695229 & LG11 & $\begin{array}{c}31138143 . .3114 \\
0131\end{array}$ & 3 & $\begin{array}{c}X P \_0034503 \\
17.1\end{array}$ & 272 \\
\hline $\begin{array}{l}\text { Bcl-2 } \\
\text { homologs }\end{array}$ & $\begin{array}{l}\mathrm{BCl}-2 \\
\text { homologs }\end{array}$ & $\mathrm{Bcl} 2 \mathrm{a}$ & 100710191 & LG18 & $\begin{array}{c}16802357 . .1682 \\
8801\end{array}$ & 2 & $\begin{array}{c}\text { XP_0034379 } \\
50.1\end{array}$ & 228 \\
\hline & & $\mathrm{Bcl} 2 \mathrm{~b}$ & 102079353 & LG9 & $\begin{array}{c}2983686 . .29912 \\
28\end{array}$ & 3 & $\begin{array}{c}\text { XP_0054619 } \\
25.1\end{array}$ & 198 \\
\hline & & $\begin{array}{l}\mathrm{Bcl} 2 \mathrm{~L} 10 \\
(\mathrm{NR} 13)\end{array}$ & 100699619 & LG1 & $\begin{array}{c}28554301 . .2855 \\
9035\end{array}$ & 3 & $\begin{array}{c}X P \_0034377 \\
47.1\end{array}$ & 231 \\
\hline & $\begin{array}{l}\text { Pro- } \\
\text { apoptotic }\end{array}$ & Baxa & 100706164 & LG1 & $\begin{array}{c}4175914 . .41848 \\
84\end{array}$ & 6 & $\begin{array}{c}X P \_0034566 \\
06.1\end{array}$ & 203 \\
\hline
\end{tabular}

The Israeli Journal of Aquaculture - Bamidgeh • IJA.73.2021.1119154 
Yang et al.

\begin{tabular}{|c|c|c|c|c|c|c|c|c|}
\hline & \multirow[t]{5}{*}{$\begin{array}{l}\text { Bcl-2 } \\
\text { homologs }\end{array}$} & Baxb & 100705633 & LG4 & $\begin{array}{c}36738199 . .3674 \\
3862\end{array}$ & 4 & $\begin{array}{c}\text { XP_0034569 } \\
32.3\end{array}$ & 192 \\
\hline & & Baxc & 109201890 & LG4 & $\begin{array}{c}25766522 . .2576 \\
9663\end{array}$ & 6 & $\begin{array}{c}X P \_0192132 \\
95.1\end{array}$ & 192 \\
\hline & & Boka & 100689837 & LG23 & $\begin{array}{c}32302318 . .3231 \\
4996\end{array}$ & 5 & $\begin{array}{c}X P \_0034443 \\
15.1\end{array}$ & 210 \\
\hline & & Bokb & 100707238 & LG18 & $\begin{array}{c}16036504 . .1604 \\
7466\end{array}$ & 5 & $\begin{array}{c}X P \_0054763 \\
55.1\end{array}$ & 213 \\
\hline & & $\mathrm{Bcl}-2 \mathrm{I} 12$ & 106097209 & LG4 & $\begin{array}{c}6538804 . .65488 \\
08\end{array}$ & 8 & $\begin{array}{c}X P \_0192114 \\
81.1\end{array}$ & 359 \\
\hline & \multirow{5}{*}{$\begin{array}{l}\text { Divergent } \\
\mathrm{Bcl}-2 \\
\text { homologs }\end{array}$} & $\mathrm{Bcl} 2 \mid 13$ & 100692352 & LG7 & $\begin{array}{c}61926215 . .6194 \\
5421\end{array}$ & 7 & $\begin{array}{c}X P \_0034471 \\
61.1\end{array}$ & 554 \\
\hline & & $\mathrm{Bcl} 2 \mathrm{|} 14$ & 102080759 & LG7 & $\begin{array}{c}1962348 . .19644 \\
34\end{array}$ & 7 & $\begin{array}{c}X P \_0054702 \\
68.1\end{array}$ & 127 \\
\hline & & $\mathrm{BCl} 2 \mathrm{|} 15^{2}$ & $\begin{array}{c}\text { I3КT992/112 } \\
846943\end{array}$ & LG5 & $\begin{array}{c}12614027 . .1261 \\
5836\end{array}$ & 3 & $\begin{array}{c}\text { I3KT99/XP_0 } \\
25763155.1\end{array}$ & 162 \\
\hline & & Bad & 100712130 & LG3 & $\begin{array}{c}10197446 . .1020 \\
2008\end{array}$ & 4 & $\begin{array}{c}X P \_0034524 \\
65.1\end{array}$ & 158 \\
\hline & & Bid & 100699927 & LG7 & $\begin{array}{c}61923921 . .6192 \\
6616\end{array}$ & 4 & $\begin{array}{c}X P \_0034471 \\
06.1\end{array}$ & 194 \\
\hline & \multirow[t]{4}{*}{$\begin{array}{l}\text { Canonical } \\
\text { BH3-only } \\
1\end{array}$} & $\mathrm{Bcl}-2 \mathrm{I} 11$ & 102081903 & LG13 & $\begin{array}{c}9890853 . .99210 \\
02\end{array}$ & 4 & $\begin{array}{c}\text { XP_0054744 } \\
58.1\end{array}$ & 223 \\
\hline & & Bmfa & 102081386 & LG19 & $\begin{array}{c}9103359 . .91152 \\
60\end{array}$ & 4 & $\begin{array}{c}X P \_0054533 \\
60.1\end{array}$ & 163 \\
\hline \multirow[t]{5}{*}{ BH3-only } & & $\mathrm{Bmfb}$ & 100690873 & LG15 & $\begin{array}{c}8978141 . .89898 \\
86\end{array}$ & 4 & $\begin{array}{c}X P \_0034556 \\
60.1\end{array}$ & 179 \\
\hline & & Bnip3a & 100697476 & LG13 & $\begin{array}{c}14388730 . .1439 \\
2585\end{array}$ & 6 & $\begin{array}{c}X P \_0034383 \\
23.1\end{array}$ & 189 \\
\hline & \multirow{3}{*}{$\begin{array}{l}\text { Other } \\
\mathrm{BH} 3 \text {-only } \\
1\end{array}$} & Bnip3b & 100705876 & LG7 & $\begin{array}{c}16871909 . .1687 \\
8512\end{array}$ & 6 & $\begin{array}{c}X P \_0054562 \\
74.1\end{array}$ & 181 \\
\hline & & Bnip3lb & 100711031 & LG12 & $\begin{array}{c}12383733 . .1238 \\
7976\end{array}$ & 6 & $\begin{array}{c}X P \_0034457 \\
27.1\end{array}$ & 236 \\
\hline & & Bnip3la & 100689723 & LG7 & $\begin{array}{c}29889572 . .2990 \\
1785\end{array}$ & 6 & $\begin{array}{c}X P \_0131239 \\
66.1\end{array}$ & 217 \\
\hline
\end{tabular}

${ }^{1}$ Canonical: the protein functional domains of these genes had only a canonical $\mathrm{BH} 3$ domain. Other: the protein functional domains of these genes had BH3 domain and TM domain.

${ }^{2}$ This gene was identified via protein search (it was totally missed by NCBI annotation pipeline).

Table 3 Classification of $\mathrm{BCl}-2$ family genes in 4 vertebrate genomes (modified from HGNC).

\begin{tabular}{|c|c|c|c|c|c|c|c|}
\hline Subsets & & Approved Symbol & Description & $\mathrm{ON}^{1}$ & $\mathrm{DR}^{1}$ & $\mathrm{CC}^{1}$ & $\mathrm{MM}^{1}$ \\
\hline \multirow{7}{*}{$\begin{array}{l}B C l-2 \\
\text { homologs }\end{array}$} & \multirow{4}{*}{$\begin{array}{l}\text { Pro-apoptotic } \\
\mathrm{BCl}-2 \text { homologs }\end{array}$} & Bax & $\begin{array}{l}\mathrm{BCL2} \text { associated } \mathrm{X} \text {, apoptosis } \\
\text { regulator }\end{array}$ & $3(4) *$ & 3 & 7 & 2 \\
\hline & & Bak1 & BCL2 antagonist/killer 1 & 0 & 0 & 0 & 1 \\
\hline & & Bok & $\begin{array}{l}\mathrm{BOK}, \mathrm{BCL} 2 \text { family apoptosis } \\
\text { regulator }\end{array}$ & 2 & 2 & 3 & 1 \\
\hline & & $\mathrm{BCl} 2 \mathrm{~L} 16^{2}$ & BCL2 like 16 & 0 & 1 & 1 & 0 \\
\hline & \multirow{3}{*}{$\begin{array}{l}\text { Anti-apoptotic } \\
B C l-2 \text { homologs }\end{array}$} & $\mathrm{Bcl} 2$ & $\mathrm{BCL} 2$, apoptosis regulator & 2 & 2 & 2 & 2 \\
\hline & & $\mathrm{Bcl} 2 \mathrm{I} 1$ & BCL2 like 1 & 2 & 2 & 1 & 2 \\
\hline & & $\mathrm{Bcl} 2 \mathrm{I} 2$ & BCL2 like 2 & 0 & 0 & 0 & 1 \\
\hline
\end{tabular}

The Israeli Journal of Aquaculture - Bamidgeh • IJA.73.2021.1119154 


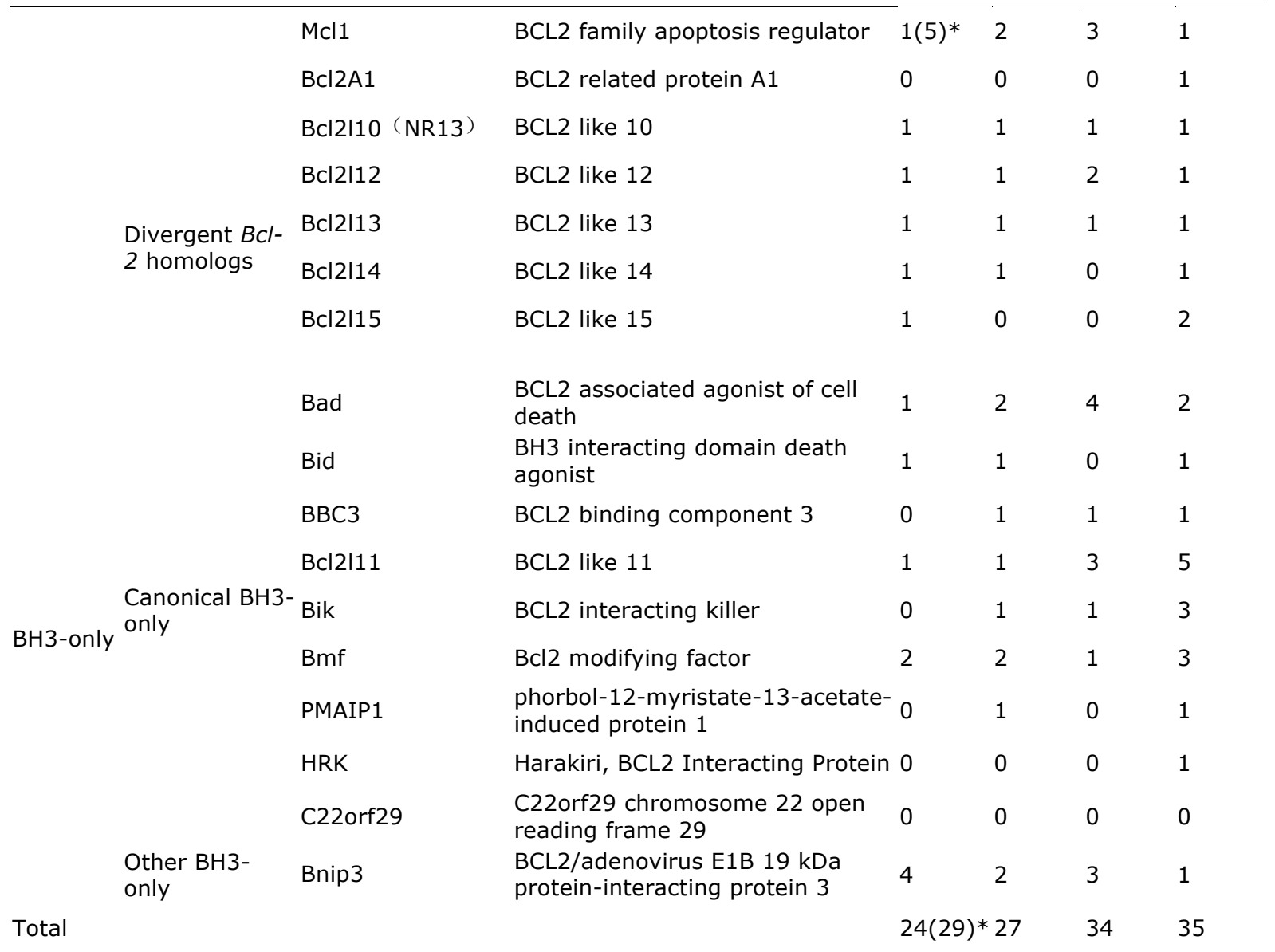

${ }^{1}$ The numbers of genes found in ON: Tilapia (Oreochromis niloticus); DR: Zebra fish (Danio rerio); CC: Common carp (Cyprinus carpio); MM: House mouse (Mus musculus).

${ }^{2} \mathrm{Bcl}-\mathrm{WAV}$, also named as Bcl2L16, was found in fish first.

*The number within parenthesis is from original bioinformatics analysis; the number outside parenthesis is based on PCR validation results.

Table 4 Structure and functional domains analysis of $\mathrm{BCl}-2$ genes family protein in tilapia.

\begin{tabular}{|c|c|c|c|c|}
\hline & groups & $\begin{array}{l}\text { Gene Name } \\
\text { assigned }\end{array}$ & 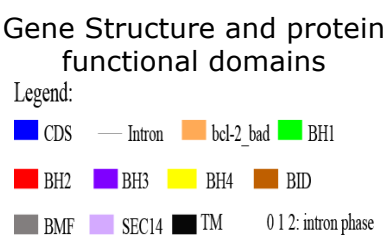 & $\begin{array}{c}\text { Predicted } \\
\mathrm{BH} 1 / 2 / 3 / 4\end{array}$ \\
\hline \multirow{12}{*}{$\begin{array}{c}B C l-2 \\
\text { homologs }\end{array}$} & \multirow{7}{*}{$\begin{array}{l}\text { Anti-apoptotic } \\
\mathrm{BCl}-2 \text { homologs }\end{array}$} & $\mathrm{Bcl} 2 \mathrm{I} 1 \mathrm{a}$ & $=0$ & $\mathrm{BH} 1 / 2 / 3$ \\
\hline & & $\mathrm{Bcl} 2 \mathrm{I} 1 \mathrm{~b}$ & 1 & $\mathrm{BH} 1 / 2 / 3 / 4$ \\
\hline & & Mcl1 & 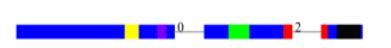 & $\mathrm{BH} 1 / 2 / 3 / 4$ \\
\hline & & $\mathrm{Bcl} 2 \mathrm{a}$ & $\mathbf{E}^{0}$ & $\mathrm{BH} 1 / 2 / 3$ \\
\hline & & $\mathrm{Bcl} 2 \mathrm{~b}$ & $\square^{2}$ & $\mathrm{BH} 2 / 4$ \\
\hline & & $\begin{array}{c}\text { BCL2L10(NR1 } \\
3 \text { ) }\end{array}$ & & $\mathrm{BH} 1 / 2 / 3 / 4$ \\
\hline & & Baxa & 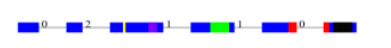 & $\mathrm{BH} 1 / 2 / 3 / 4$ \\
\hline & \multirow{5}{*}{$\begin{array}{l}\text { Pro-apoptotic } \\
\text { BCl-2 homologs }\end{array}$} & Baxb & $\square \square^{0}=\square^{0}$ & $\mathrm{BH} 1 / 2 / 3$ \\
\hline & & Baxc & $\square^{2}=U^{\prime}=$ & $\mathrm{BH} 1 / 2 / 3 / 4$ \\
\hline & & Boka & $\square \mathbf{a}^{0} \mathbf{a}^{2} \mathbf{u}^{2} \mathbf{m}$ & $\mathrm{BH} 1 / 2 / 3 / 4$ \\
\hline & & Bokb & $=-$ & $\mathrm{BH} 1 / 2 / 3 / 4$ \\
\hline & & $\mathrm{Bcl} 2 \mathrm{I} 12$ & $-\square---\square$ & $\mathrm{BH} 2$ \\
\hline
\end{tabular}




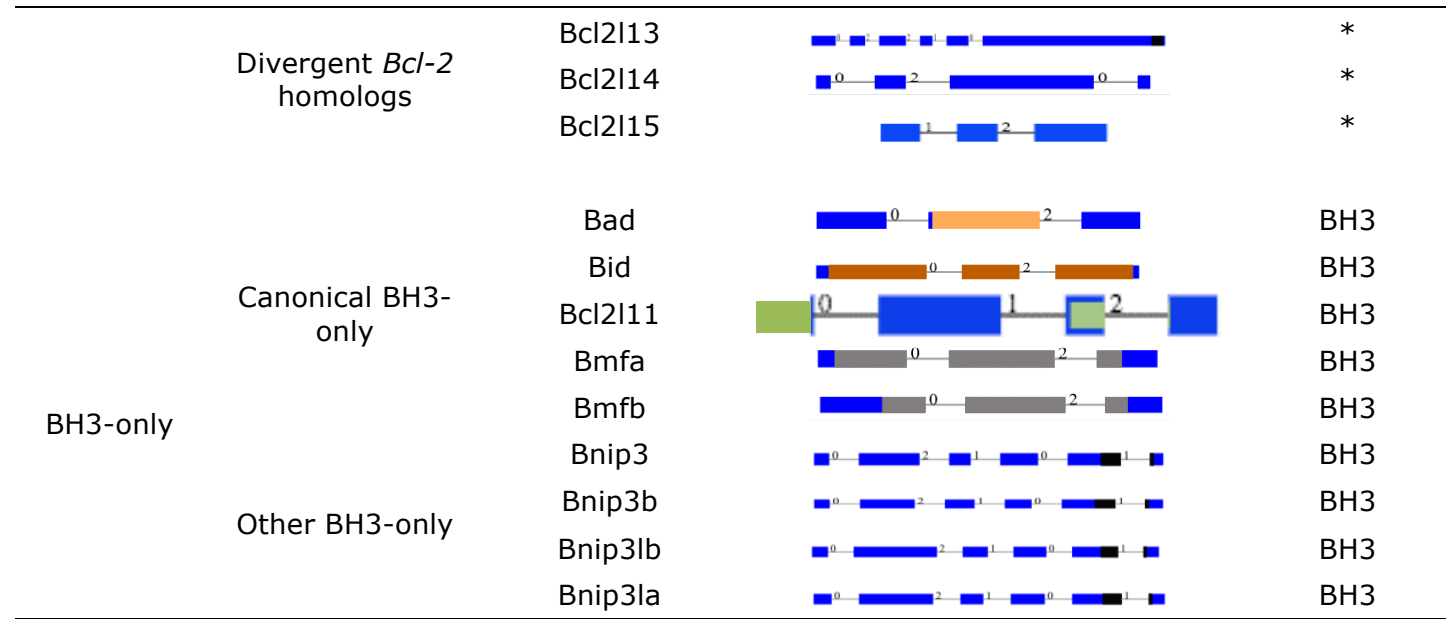

* Not specifically for each $\mathrm{BH}$ domain. But combined domain search shows it contains the BCL-2-like region spanning BH1-4.

Chromosomal location and intron analysis of $\mathrm{BCl}-2$ family genes in tilapia

Base on the genomic distribution analysis of $\mathrm{BCl}-2$ family genes, the results showed that the genes of the 24 family members were widely distributed on 16 different chromosomes, and their distribution appeared to be uneven (Table $\mathbf{2}$ ).

The analysis of intron number showed that the number of introns in the same subgroup members of $\mathrm{BCl}-2$ family had little difference, while the number of introns in different subgroup had obvious difference. Among them, the divergent $\mathrm{BCl}-2$ homologs subgroup members and pro-apoptotic subgroup members had more the average number of introns, was 4.8 and 4.2, respectively. Moreover, the number of introns in divergent subgroup members is quite different. The average number of introns in anti-apoptotic subgroup, canonical BH3-only subgroup and the other BH3-only subgroup was 1.8, 3, and 2.2, respectively (Table $\mathbf{2}$ ).

Phylogenetic analysis of BCL-2 family protein in tilapia and other selected Animals

We further constructed $\mathrm{BCl}-2$ gene phylogenetic trees among the tilapia, two other selected teleost (zebrafish (D. rerio) and common carp (C. carpio)) and mammal (house mouse (M. musculus)) using Neighbor-Joining method (Figure 1). All BCl-2 genes were grouped into seven groups in the phylogenetic tree, which was like the classification results based on conservative domain. The anti-apoptotic subgroup and pro-apoptotic subgroup of $\mathrm{BCl}-2$ homologs genes subfamily were clustered on the same branches, respectively. Interestingly, the member of divergent subgroup of $\mathrm{BCl}-2$ homologs genes subfamily and BH3-only subfamily had a closer evolution relationship. Most of the BH3-only subfamily members were grouped into the same branch. However, a small number of $\mathrm{BH}$-only genes were clustered into branches of the other $\mathrm{BCl}-2$ subfamilies, and these genes were from multiple vertebrate species. 


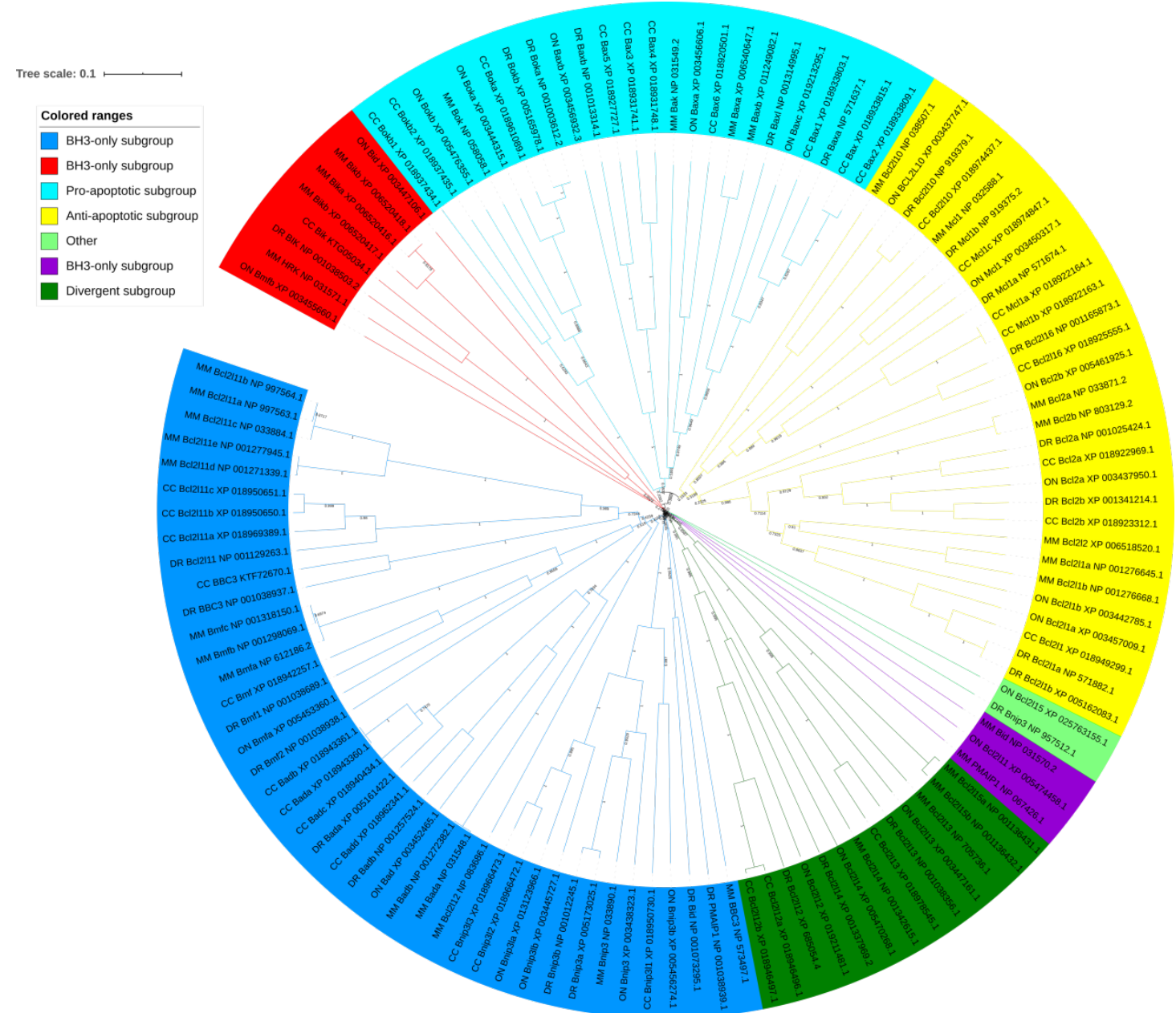

Figure 1 Molecular phylogeny for 120 BCL-2 family genes members identified in three bony fish genomes and Mus musculus genomes in silico. The bootstrap scores with 1000 trials are shown on branches. ON: Tilapia (Oreochromis niloticus); DR: Zebra fish (Danio rerio); CC: Common carp (Cyprinus carpio); MM: House mouse (Mus musculus)

Expression profiling of BCL-2 family genes in tilapia under normal temperature and low temperature stress

The heatmap was constructed to cluster tilapia $\mathrm{BCl}-2$ family genes with same expression pattern under different temperature conditions (Figure 2). Genes from the same subfamily revealed different expression patterns at different temperatures, and no correlation could be identified among expression patterns of genes from different subfamilies. Notably, except in the muscle, at $10^{\circ} \mathrm{C}$, the transcriptional expression levels of almost all canonical $\mathrm{BH} 3-$ only subgroup genes were lower than $30^{\circ} \mathrm{C}$. On the contrary, in every tissue, at $10^{\circ} \mathrm{C}$, the transcriptional expression levels of almost all other $\mathrm{BH} 3$-only subgroup members were higher than $30^{\circ} \mathrm{C}$. At $10^{\circ} \mathrm{C}$, the transcriptional expression levels of most of anti-apoptotic $\mathrm{BCl}-2$ homologs subgroup members were higher than $30^{\circ} \mathrm{C}$, except in liver and fin. At $10^{\circ} \mathrm{C}$, the transcriptional expression levels of most of pro-apoptotic $\mathrm{BCl}-2$ homologs subgroup members were lower than $30^{\circ} \mathrm{C}$, except in intestine, muscle, brain and heart. At $10^{\circ} \mathrm{C}$, the transcriptional expression levels of most of divergent $B C l-2$ homologs subgroup members were lower than $30^{\circ} \mathrm{C}$, except in muscle and brain. 


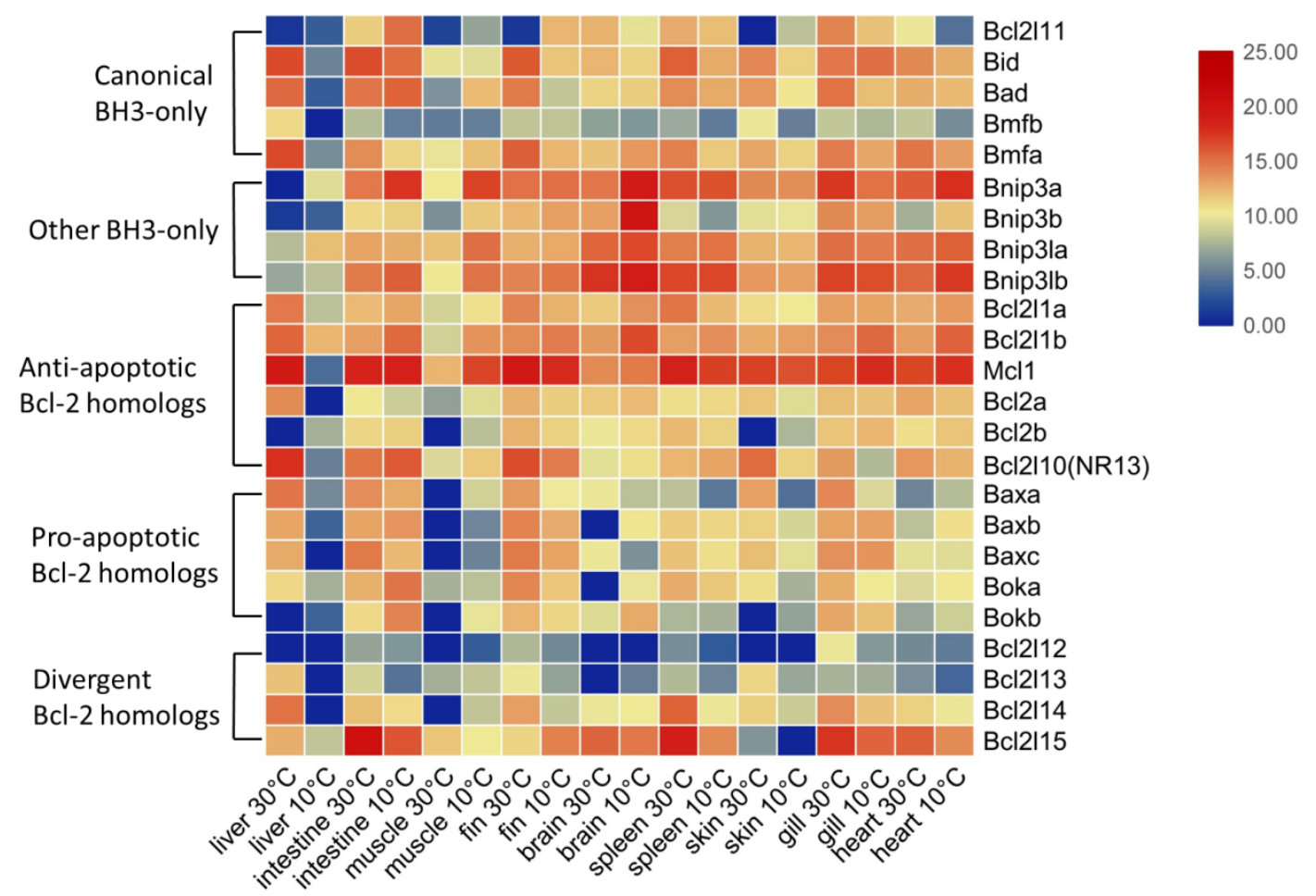

Figure 2 The expression profile of spleen BCL-2 family genes at normal temperature $\left(30{ }^{\circ} \mathrm{C}\right)$ and low temperature $\left(10{ }^{\circ} \mathrm{C}\right)$ stresses conditions. Control with the Bcl2l11 expression in liver at $30{ }^{\circ} \mathrm{C}$, then the $\log _{2}$ values of all genes relative to its expression were used for the heatmap.

\section{Discussion}

As reported, the $\mathrm{BCl}-2$ gene family has been discovered and grouped into three categories according to their functions (Suzanne and Adams, 2002). (1) The anti-apoptotic protein subfamily, including BCL-xL (SW et al., 1996), BCL-2 (Petros et al., 2001), BCL-W (also known as Bcl-2l10) (Denisov et al., 2003; Hinds et al., 2014), MCL-1 (myeloid cell leukemia sequence 1) (Day et al., 2005), and Al (also known as BFLI) (Herman et al., 2008). These genes contain 4-short conserved $\mathrm{BH}$ domains (BH1-BH4) (Roy et al., 2014) and a Cterminal hydrophobic tail structure, ie, transmembrane (TM) function domain. The TM junction domain allows them to localize in the mitochondrial outer membrane, and occasionally to the surface of the endoplasmic reticulum. The transmembrane structure of the protein is oriented toward the cytoplasm (Akao et al., 1994; Krajewski et al., 1993; Nguyen et al., 1993). These members can block apoptosis by inhibiting their pro-apoptotic counterparts (Adams and Cory, 2007); (2) the pro-apoptotic protein subfamily, including BAX (BCL-2 homologous antagonist/killer protein), BAK (BCL-2-associated $X$ protein), and BOK containing multi-domains and directly facilitate MOMP (Czabotar et al., 2014; Youle and Strasser, 2008). They are structurally similar to the former members and contain all domains except BH4 (Tsujimoto et al., 1985); (3) The BH3-only protein subfamily, a special pro-apoptotic protein group that only has $\mathrm{BH} 3$ domain. It includes $\mathrm{BIM}$ (BCL-2 interacting mediator of cell death), BID, BAD (BCL-2-associated death promoter protein), PUMA ( $p 53$ up-regulated modulator of apoptosis protein) and NoxA. They act as initiators in response to discrete cellular apoptotic stimuli (such as growth factor withdrawal, DNA damage, and anoikis) (Czabotar et al., 2014; Yoshihide, 2010).

So far, there had been no report on the identification and analysis of $\mathrm{BCl}-2$ family genes at the genome-wide level in teleosts. However, the tilapia genome and transcriptome data sets facilitated the structural and functional analysis of $\mathrm{BCl}-2$ family at the genome-wide level. In this study, a total of $24 \mathrm{BCl}-2$ family genes were identified in the tilapia genome. And referring to the aforementioned classification, these $\mathrm{BCl}-2$ family genes of tilapia were divided into two subfamilies, $\mathrm{BCl}-2$ homologs and $\mathrm{BH} 3-$ only, based on the composition of $\mathrm{BH}$ motifs. The subfamily of $\mathrm{BCl}-2$ homologs contained anti-apoptotic $\mathrm{Bcl}-2$ subgroup, Pro- 
apoptotic $\mathrm{BCl}-2$ subgroup and Divergent $\mathrm{BCl}-2$ subgroup. $\mathrm{BH}$-only subfamily contained a canonical $\mathrm{BH} 3$-only subgroup and another $\mathrm{BH} 3$-only subgroup.

The phylogenetic analysis of the homologous sequences of $\mathrm{BCl}-2$ family genes of tilapia and other species (two other teleost and a mammal species) suggested that the $\mathrm{BCl}-2$ family genes did not aggregate by species, demonstrating sequence conservation of different types of $\mathrm{BCl}-2$ family members. Moreover, the Anti-apoptotic, Pro-apoptotic, and Divergent subgroup from $\mathrm{BCl}-2$ homologs subfamily genes were respectively clustered as three branches, and Divergent $\mathrm{BCl}-2$ homologs subgroup and $\mathrm{BH} 3$-only subfamily had revealed a closer evolutionary relationship. According to this result and referring to the domain analysis of $\mathrm{BCl}-2$ family proteins, we reclassified $\mathrm{BCl}-2$ family genes into two subfamilies, $\mathrm{BCl}-2$ homologs subfamily (including Anti-apoptotic $\mathrm{BCl}-2$ homologs and Proapoptotic $\mathrm{BCl}-2$ homologs), and other $\mathrm{BCl}-2$ subfamily. The above mentioned results have further indicated that there was a clear differentiation between these two subfamilies, which evolved independently of each other. In addition, the results of domain analysis of $\mathrm{BCl}-2$ gene family indicated that genes from the same subfamily was highly conserved among different species.

Gene expression profiling showed that upon low temperature conditions $\left(10^{\circ} \mathrm{C}\right)$, the transcriptional expression of most of the anti-apoptotic $\mathrm{BCl}-2$ homologs subgroup genes in most tissues of tilapia (except in liver and fin), was higher than those at normal temperature conditions $\left(30^{\circ} \mathrm{C}\right)$. As for two types of typical apoptosis-promoting genes including pro-apoptotic and canonical $\mathrm{BH} 3$-only genes, the transcriptional expression of most of typical apoptosis-promoting genes at low temperature conditions $\left(10^{\circ} \mathrm{C}\right)$ was lower when comparing with those at normal temperature conditions $\left(30^{\circ} \mathrm{C}\right)$. The results indicated that low temperature had a significant effect on tilapia, overexpression of anti-apoptotic genes in tilapia under low temperature stress conditions formed apoptosis resistance (Mcdonnell et al., 1989). Explanation for this may be that anti-apoptotic proteins can binds to pro-apoptotic proteins. Meanwhile, the body can resist the low-temperature stress and maintain the body function, by increasing the expression of anti-apoptosis, organism inhibited apoptosis (Chipuk and Green, 2008; Dewson et al., 2012; Dewson et al., 2008; Green and Levine, 2014; Haiming et al., 2011).In addition, apoptosis-promoting genes also include other $\mathrm{BH} 3$-only genes, the transcriptional expression of the other $\mathrm{BH}$-only genes in every organization of tilapia at low temperature conditions $\left(10^{\circ} \mathrm{C}\right)$ was higher than those at $30^{\circ} \mathrm{C}$, the reason may be that the apoptosis promoting protein BNIP3la, BNIP3a, BNIP3b and BNIP3Ib and the anti-apoptotic BCL-2 protein molecules could form a heterodimer to promote apoptosis (Kelekar and Thompson, 1998). Comparative analysis also revealed that hypothermia had a significant induced effect on apoptotic.

\section{Conclusion}

In the present study, we identified and characterized $24 \mathrm{BCl}-2$ family genes in tilapia. A phylogenetic analysis of the tilapia $\mathrm{BCl}-2$ family genes indicated evolutionary conservation and diversification. Large scale-based expression profiling showed that low temperature stress has a greater impact on the expression of $\mathrm{BCl}-2$ family genes in the brain, muscle, heart, and gill than those in the intestine and other tested tissues of tilapia. The information generated in this study will facilitate further research on $\mathrm{BCl}-2$ genes and other gene families in tilapia.

\section{Acknowledgements}

This work was supported by the National Natural Science Foundation of China (31402290) and the China Agriculture Research System (Grant No. CARS-46).

\section{References}

Adams, J. M., and Cory, S, 2007. The Bcl-2 apoptotic switch in cancer development and therapy. Oncogene, 26(9), 1324-1337. doi:10.1038/sj.onc.1210220.

Adams, J. M., and Cory, S, 2018. The BCL-2 arbiters of apoptosis and their growing role as cancer targets. Cell Death and Differentiation, 25(1), 27-36. doi: 10.1038/cdd.2017.161. 
Akao, Y., Otsuki, Y., Kataoka, S., Ito, Y., and Tsujimoto, Y, 1994. Multiple subcellular localization of bcl-2: detection in nuclear outer membrane, endoplasmic reticulum membrane, and mitochondrial membranes. Cancer Research, 54(9), 2468.

Aouacheria, A., Laval, V. R. d., Combet, C., and Hardwick, J. M, 2013. Evolution of Bcl-2 homology motifs: homology versus homoplasy. Trends in Cell Biology, 23(3), 103111. doi:10.1016/j.tcb.2012.10.010.

Atwood, H. L., Tomasso, J. R., Webb, K., and Gatlin, D. M, 2015. Low-temperature tolerance of Nile tilapia, Oreochromis niloticus: effects of environmental and dietary factors. Aquaculture Research, 34(3), 241-251. doi: 10.1046/j.1365-2109.2003.00811.x.

Behrends, L. L., and Smitherman, R. O, 2010. Development of a cold-tolerant population of red tilapia through introgressive hybridization. Journal of the World Aquaculture Society, 15(1-4), 172-178. doi:10.1111/j.1749-7345.1984.tb00150.x.

Borner, C, 2003. The Bcl-2 protein family: sensors and checkpoints for life-or-death decisions. Molecular Immunology, 39(11), 0-647. doi:10.1016/s0161-5890(02)00252-3.

Chipuk, J. E., and Green, D. R, 2008. How do BCL-2 proteins induce mitochondrial outer membrane permeabilization? Trends in Cell Biology, 18(4), 157-164. doi:10.1016/j.tcb.2008.01.007.

Christoph, B, 2003. The $\mathrm{Bcl}-2$ protein family: sensors and checkpoints for life-or-death decisions. Molecular Immunology, 39(11), 0-647. doi:10.1016/S0161-5890(02)00252-3

Czabotar, P. E., Lessene, G., Strasser, A., and Adams, J. M, 2014. Control of apoptosis by the BCL-2 protein family: implications for physiology and therapy. Nat Rev Mol Cell Biol, 15(1), 49-63. doi: $10.1038 / \mathrm{nrm} 3722$.

Danial, N. N., and Korsmeyer, S. J, 2004. Cell death: critical control points. Cell, 116(2), 205-219. doi:10.1016/S0092-8674(04)00046-7.

Day, C. L., Chen, L., Richardson, S. J., Harrison, P. J., Huang, D. C., and Hinds, M. G, 2005. Solution structure of prosurvival $\mathrm{Mcl}-1$ and characterization of its binding by proapoptotic BH3-only ligands. Journal of Biological Chemistry, 280(6), 4738-4744. doi: 10.1074/jbc.M411434200.

Denisov, A. Y., Madiraju, M. S., Chen, G., Khadir, A., Beauparlant, P., Attardo, G., Shore, G. C., and Gehring, K, 2003. Solution structure of human BCL-w: modulation of ligand binding by the C-terminal helix. Journal of Biological Chemistry, 278(23), 2112421128. doi:10.1074/jbc.M301798200.

Dewson, G., Ma, S., Frederick, P., Hockings, C., Tan, I., Kratina, T., and Kluck, R. M, 2012. Bax dimerizes via a symmetric BH3:groove interface during apoptosis. Cell Death and Differentiation, 19(4), 661-670. doi:10.1038/cdd.2011.138.

Dewson, G., Kratina, T., Sim, H. W., Puthalakath, H., Adams, J. M., Colman, P. M., and Kluck, R. M, 2008. To Trigger Apoptosis, Bak Exposes Its BH3 Domain and Homodimerizes via BH3:Groove Interactions. Molecular Cell, 30(3), 369-380. doi:10.1016/j.molcel.2008.04.005.

Green, D. R., and Levine, B, 2014. To be or not to be? How selective autophagy and cell death govern cell fate. Cell, 157(1), 65-75. doi:10.1016/j.cell.2014.02.049.

Haiming, D., Alyson, S., X Wei, M., Schneider, P. A., Yuan-Ping, P., and Kaufmann, S. $\mathbf{H}, 2011$. Transient binding of an activator $\mathrm{BH} 3$ domain to the Bak $\mathrm{BH} 3$-binding groove initiates Bak oligomerization. Journal of Cell Biology, 194(1), 39-48. doi: $10.1083 /$ jcb.201102027.

Herman, M. D., Nyman, T., Welin, M., Lehtiö, L., Flodin, S., Trésaugues, L., Kotenyova, T., Flores, A., and Nordlund, $\mathbf{P}, 2008$. Completing the family portrait of the anti-apoptotic $\mathrm{Bcl}-2$ proteins: crystal structure of human Bfl-1 in complex with Bim. Febs Letters, 582(25), 3590-3594. doi:10.1016/j.febslet.2008.09.028

Hinds, M. G., Lackmann, M., Skea, G. L., Harrison, P. J., Huang, D. C., and Day, C. $\mathbf{L}, 2014$. The structure of $\mathrm{Bcl}-\mathrm{w}$ reveals a role for the C-terminal residues in modulating biological activity. Embo Journal, 22(7), 1497-1507. doi:10.1093/emboj/cdg144.

Kelekar, A., and Thompson, C. B, 1998. Bcl-2-family proteins: the role of the $\mathrm{BH} 3$ domain in apoptosis. Trends in Cell Biology, 8(8), 324-330. doi:10.1016/s09628924(98)01321-x. 
Kindle, K. R., and Whitmore, D. H, 2010. Biochemical indicators of thermal stress in Tilapia aurea (Steindachner). Journal of Fish Biology, 29(2), 243-255. doi:10.1111/j.10958649.1986.tb04942.x.

Krajewski, S., Tanaka, S., Takayama, S., Schibler, M. J., Fenton, W., and Reed, J. C, 1993. Investigation of the subcellular distribution of the bcl-2 oncoprotein: residence in the nuclear envelope, endoplasmic reticulum, and outer mitochondrial membranes. Cancer Research, 53(19), 4701-4714.

Kvansakul, M., Yang, H., Fairlie, W., Czabotar, P. E., Fischer, S. F., Perugini, M., Huang, D., Colman, P.M, 2008. Vaccinia virus anti-apoptotic F1L is a novel Bcl-2-like domain-swapped dimer that binds a highly selective subset of $\mathrm{BH}$-containing death ligands. Cell Death and Differentiation, 15(10), 1564. doi:10.1038/cdd.2008.83.

Lanave, C., Santamaria, M., and Saccone, C, 2004. Comparative genomics: the evolutionary history of the Bcl-2 family. Gene, 333(25), 71-79. doi:10.1016/j.gene.2004.02.017.

Levine, B., Sinha, S. C., and Kroemer, G, 2008. Bcl-2 family members: Dual regulators of apoptosis and autophagy. Autophagy, 4(5), 600-606. doi:10.4161/auto.6260.

Li, S. S., Li, C. C., Dey, M., Gagalac, F., and Dunham, R, 2002. Cold tolerance of three strains of Nile tilapia, Oreochromis niloticus, in China. Aquaculture, 213(1), 123-129. doi:10.1016/S0044-8486(02)00068-6.

Los, D. A., and Murata, N, 2004. Membrane fluidity and its roles in the perception of environmental signals. Biochim Biophys Acta, 1666(1), 142-157. doi:10.1016/j.bbamem.2004.08.002.

Mcdonnell, T. J., Deane, N., Platt, F. M., Nunez, G., Jaeger, U., Mckearn, J. P., Korsmeyer, and J., S, 1989. bcl-2-immunoglobulin transgenic mice demonstrate extended B cell survival and follicular lymphoproliferation. Cell, 57(1), 79-88. doi:10.1016/0092-8674(89)90174-8.

Muchmore, S. W., Sattler, M., Liang, H., Meadows, R. P., Harlan, J. E., Yoon, H. S., Nettesheim, D., Chang, B. S., Thompson, C. B., Wong, S. L., Ng, S. L., and Fesik, S. W, 1996. X-ray and NMR structure of human Bcl-xL, an inhibitor of programmed cell death. Nature, 381(6580), 335-341. doi:10.1038/381335a0.

Nguyen, M., Millar, D. G., Yong, V. W., Korsmeyer, S. J., and Shore, G. C, 1993. Targeting of $\mathrm{BCl}-2$ to the mitochondrial outer membrane by a $\mathrm{COOH}$-terminal signal anchor sequence. Journal of Biological Chemistry, 268(34), 25265-25268.

Petros, A. M., Medek, A., Nettesheim, D. G., Kim, D. H., Yoon, H. S., Swift, K., Matayoshi, E. D., Oltersdorf, T., and Fesik, S. W, 2001. Solution structure of the antiapoptotic protein bcl-2. Proceedings of the National Academy of Sciences of the United States of America, 98(6), 3012-3017. doi:10.1073/pnas.041619798.

Potts, W. T., Foster, M. A., Rudy, P. P., and Howells, G. P, 1967. Sodium and water balance in the cichlid teleost, Tilapia mossambica. Journal of Experimental Biology, 47(3), 461.

Renault, T. T., and Chipuk, J. E, 2014. Death upon a kiss: mitochondrial outer membrane composition and organelle communication govern sensitivity to BAK/BAX-dependent apoptosis. Chemistry and Biology, 21(1), 114-123. doi:10.1016/j.chembiol.2013.10.009.

Roy, M. J., Vom, A., Czabotar, P. E., and Lessene, G, 2014. Cell death and the mitochondria: therapeutic targeting of the BCL-2 family-driven pathway. British Journal of Pharmacology, 171(8), 1973-1987. doi:10.1111/bph.12431

Sattler, M., Liang, H., Nettesheim, D., Meadows, R. P., Harlan, J. E., Eberstadt, M., Yoon, H. S., Shuker, S. B., Chang, B. S., and Minn, A. J, 1997. Structure of Bcl-XL-Bak peptide complex: recognition between regulators of apoptosis. Science, 275(5302), 983986. doi: $10.1126 /$ science.275.5302.983

Strasser, A., Puthalakath, H., Bouillet, P., Huang, D. C., O'Connor, L., O'Reilly, L. A., Cullen, L., Cory, S., and Adams, J. M, 2010. The role of bim, a proapoptotic BH3only member of the Bcl-2 family in cell-death control. Annals of the New York Academy of Sciences, 917(1), 541-548. doi:10.1111/j.1749-6632.2000.tb05419.x 
Sun, L. T., Chen, G. R., and Chang, C. F, 1992. The physiological responses of tilapia exposed to low temperatures. Journal of Thermal Biology, 17(3), 149-153. doi:10.1016/0306-4565(92)90026-C.

Suzanne, C., and Adams, J. M, 2002. The Bcl2 family: regulators of the cellular life-ordeath switch. Nature Reviews Cancer, 2(9), 647-656. doi:10.1038/nrc883.

Suzanne, C., Huang, D. C. S., and Adams, J. M, 2003. The Bcl-2 family: roles in cell survival and oncogenesis. Oncogene, 22(53), 8590. doi:10.1038/sj.onc.1207102.

Tsujimoto, Y, 1989. Overexpression of the human BCL-2 gene product results in growth enhancement of Epstein-Barr virus-immortalized B cells. Proceedings of the National Academy of Sciences of the United States of America, 86(6), 1958-1962. doi:10.1073/pnas.86.6.1958.

Tsujimoto, Y., Finger, L. R., Yunis, J., Nowell, P. C., and Croce, C. M, 1984. Cloning of the chromosome breakpoint of neoplastic $B$ cells with the $t(14 ; 18)$ chromosome translocation. Science, 226(4678), 1097-1099. doi:10.1126/science.6093263.

Tsujimoto, Y., Jaffe, E., Cossman, J., Gorham, J., Nowell, P. C., and Croce, C. M, 1985. Clustering of breakpoints on chromosome 11 in human B-cell neoplasms with the $\mathrm{t}(11 ; 14)$ chromosome translocation. Nature, 315, 340. doi:10.1038/315340a0.

WohIfarth, G. W., and Hulata, G. I, 1981. Applied genetics of tilapias.

Yang, C., Jiang, M., Wen, H., Tian, J., Liu, W., Wu, F., and Gou, G, 2015. Analysis of differential gene expression under low-temperature stress in Nile tilapia (Oreochromis niloticus) using digital gene expression. Gene, 564(2), 134-140. doi:10.1016/j.gene.2015.01.038

Yang, C., Wu, F., Lu, X., Jiang, M., Liu, W., Yu, L., Tian, J., and Wen, H, 2017. Growth arrest specific gene 2 in tilapia (Oreochromis niloticus): molecular characterization and functional analysis under low-temperature stress. BMC Molecular Biology, 18(1), 18. doi: 10.1186/s12867-017-0095-y

Yoshihide, T, 2010. Cell death regulation by the $\mathrm{Bcl}-2$ protein family in the mitochondria. Journal of Cellular Physiology, 195(2), 158-167. doi:10.1002/jcp.10254.

Youle, R. J., and Strasser, A, 2008. The BCL-2 protein family: opposing activities that mediate cell death. Nat Rev Mol Cell Biol, 9(1), 47-59. doi:10.1038/nrm2308.

Zhou, T., Gui, L., Liu, M., Li, W., Hu, P., Duarte, D. F. C., Niu, H., and Chen, L, 2018. Transcriptomic responses to low temperature stress in the Nile tilapia, Oreochromis niloticus. Fish and Shellfish Immunology, 84, 1145-1156. doi:10.1016/j.fsi.2018.10.023. 